\title{
PARP1 Inhibition as a Novel Therapeutic Target for Keloid Disease
}

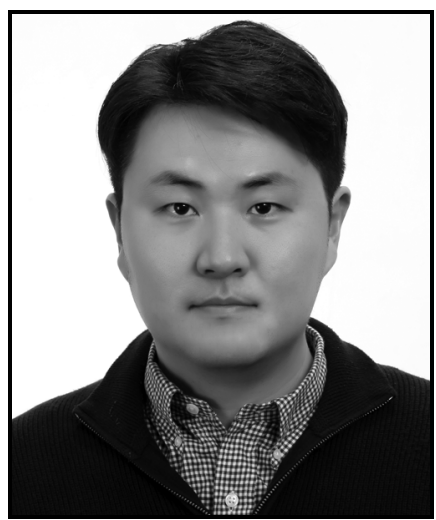

Tae Hwan Park, MD, PhD

Submitted for publication December 3, 2018 Accepted in revised form January 7, 2019.

${ }^{*}$ Correspondence: Tae Hwan Park, MD, PhD; Department of Plastic and Reconstructive Surgery, CHA Bundang Medical Center, CHA University School of Medicine, 59, Yatap-ro, Bundang-gu, Seongnam 13496, Republic of Korea (e-mail: pspark0124@cha.ac.kr; hard-piano@ hanmail.net).

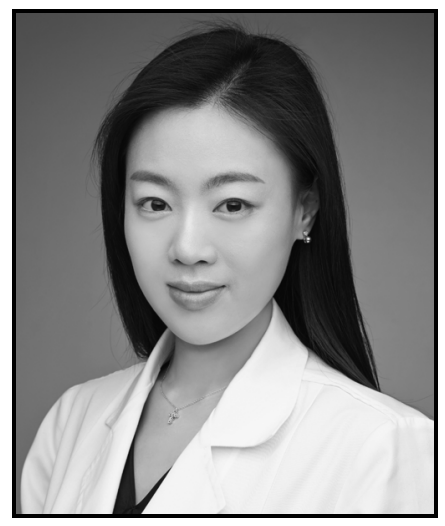

Yuri Cho, MD, PhD

*Correspondence: Department of Internal Medicine, Gangnam CHA Medical Center, CHA University School of Medicine, 569, Nonhyon-ro, Gangnam-gu, Seoul 06135, Republic of Korea (e-mail: yuricho@cha.ac.kr).

\author{
Tae Hwan Park, ${ }^{1, \#, *}$ Chan Woo Kim, ${ }^{1}$ Jin Sik Choi, ${ }^{1}$ Yun Joo Park, ${ }^{2}$ \\ Yosep Chong, ${ }^{3}$ Min Ji Park, ${ }^{4}$ and Yuri Cho ${ }^{4, \#, *}$ \\ ${ }^{1}$ Department of Plastic and Reconstructive Surgery, CHA Bundang Medical Center, CHA University School of Medicine, \\ Seongnam, Republic of Korea. \\ ${ }^{2}$ Department of Radiology, Hallym University Sacred Heart Hospital, Hallym University College of Medicine, Anyang,
Republic of Korea. \\ ${ }^{3}$ Department of Hospital Pathology, Yeouido St. Mary's Hospital, The Catholic University of Korea College of Medicine, \\ Seoul, Republic of Korea. \\ ${ }^{4}$ Department of Internal Medicine, Gangnam CHA Medical Center, CHA University School of Medicine, Seoul, Republic of Korea. \\ "These two authors contributed equally to this work.
}

Objective: Inactivation of poly(ADP-ribose) polymerase 1 (PARP1) has been found to have protective effect in several fibrotic diseases. But the effect is not studied yet in keloids. Herein, we evaluated the therapeutic effect of PARP1 inhibitor, rucaparib, for keloids.

Approach: The protein expressions of $P A R P 1$ and smad3 were evaluated with western blotting in keloids and controls. The effect of rucaparib was evaluated using 3-(4,5-dimethylthiazol-2-yl)-2,5-diphenyltetrazolium bromide assay and migration assay. We further analyzed the effect of rucaparib on patientderived keloid xenograft murine model.

Results: The protein expressions of $P A R P 1$ and smad3 were significantly higher in keloid tissue. Rucaparib $(20 \mu \mathrm{M})$ significantly suppressed the proliferation of keloid fibroblasts. Moreover, the combination of rucaparib $(20 \mu \mathrm{M})$ and triamcinolone $(50 \mu \mathrm{M})$ showed additive suppressive effect on keloid fibroblasts. Migration assay showed that rucaparib $(10 \mu \mathrm{M})$ significantly suppressed the migration of keloid fibroblasts. Fibrosis markers in keloid fibroblasts significantly decreased after rucaparib treatment $(20 \mu \mathrm{M})$. In patient-derived keloid xenograft model, rucaparib significantly reduced the size of keloid tissue.

Innovation and Conclusion: The study data suggest PARP1 might be a novel therapeutic target for keloid disease. PARP1 inhibitor, rucaparib, might be a promising therapeutic drug for the treatment of keloid disease.

Keywords: keloid, PARP1, rucaparib, fibroblast

\section{INTRODUCTION}

KELOID DISEASE, WHICH Is caused by impaired appropriate stop signals in wound healing processes, results in aberrant proliferation of dermal fibroblasts, inflammation, and excessive deposition of extracellular matrix. ${ }^{1,2}$ The term "keloid" was coined in 1806 to describe the crab claw-like appearance of the scar and is strictly defined as scars that spread beyond the boundaries of the original wound and that do not regress spontaneously. ${ }^{1,3}$ In addition to a genetic predisposition, other factors such as skin tension, deregu- (c) Tae Hwan Park et al., 2019. Published by Mary Ann Liebert, Inc. This Open Access article is distributed under the terms of the Creative Commons License (http://creativecommons.org/licenses/by/ 4.0), which permits unrestricted use, distribution, and reproduction in any medium, provided the original work is properly cited. 
lated wound healing, immune dysfunction, abnormal apoptosis, and sebaceous gland density have been implicated in the etiology of keloid scarring. These pathogenic processes are mediated by complex signal transduction cascades that form crosstalk networks between many different signaling pathways, including the transforming growth factor-beta 1 (TGF- $\beta 1$ )-Smad3 pathway, ${ }^{4-7}$ interleukin-6 (IL-6) pathway, ${ }^{8-13}$ insulin-like growth factor pathway, ${ }^{14,15}$ and mitogen-activated protein kinase pathway. ${ }^{16,17}$

The complexity of the wound-healing process and the lack of proper animal models for keloid scar formation have also restricted progress in research aimed at revealing these mechanisms. However, recent studies validated reliable humanized keloid implantation models using immunocompromised or immunocompetent animals. ${ }^{18-20}$ Considering that immune network between host and microenvironment is an important factor responsible for keloid disease, immunocompromised animal models lacking $\mathrm{T}$ cell or $\mathrm{B}$ cell immune responses may not recapitulate that of the original keloid immune response. Therefore, we adopted patient-derived keloid xenograft models with immunocompetent murine models to provide a more accurate reflection in this study. ${ }^{18}$

Poly(ADP-ribose) polymerases-1 (PARP-1) is the molecule involved in DNA repair, balance of cellular energetic pools, which culminates with necrosis and cell dysfunction, and expression of proinflammatory genes. ${ }^{21}$ In fact, overactivation of $P A R P-1$ has been found in many physiological conditions, such as inflammatory injury, which are triggered by oxidative stress and DNA damage. Recently, therapeutic usefulness of $P A R P-1$ has been implicated in several fibrotic conditions, such as liver, kidney, and lung, where PARP-1 inhibitors attenuate the disease progress and fibrotic detrimental effects. ${ }^{22-24}$

\section{CLINICAL PROBLEM ADDRESSED}

Over several decades, no single treatment option has been advocated and none of them produced consistent and effective therapeutic results, and high rates of recurrence are common after surgery alone. Many authors have proposed numerous treatment options, but none of them clearly successfully eradicated keloid disease. ${ }^{25-27}$ These findings reflect the current lack of knowledge regarding the exact molecular mechanisms and pathogenic mechanisms that underlie keloid formation. The PARP1 inhibitor, rucaparib is a recently FDA-approved therapy for ovarian cancer. In the current study, we would like to reveal the in vitro effect of pharmacological inhibition with rucaparib in terms of cell migration, proliferation, and expression of fibrosis-related markers. We further analyze its effect in vivo using patientderived keloid xenograft model.

\section{MATERIALS AND METHODS \\ Ethics approval}

Following approval from the Institutional Review Board in CHA Bundang Medical Center, which adheres to the ethical standards as formulated in the Declaration of Helsinki, keloid tissues were obtained from eight patients undergoing surgical excision after obtaining a written informed consent from all of the patients. Keloid diagnosis was made on the basis of its clinical and pathological findings.

\section{Patients}

Patients with keloids who presented to the outpatient clinic were included in the study based on the following criteria: (1) the scar was elevated and extended beyond the dimensions of the initial injury site or lesion; (2) patients were older than 18 years; (3) surgical excision was scheduled; (4) patients received no additional treatment or medication during the study and before surgical excision; and (5) patients signed up for the data use agreement as a basis to the clinical study. Patients were excluded from the study if they were unavailable for follow-up or wanted to stop treatment for any reason. Patients who had received any additional adjuvant therapy during the treatment were also excluded from the study. A total of eight keloids on nine patients were included in this study and all keloids showed deep thickness. The detailed information of the cases is listed in Table 1.

\section{Animal care}

All animal protocols used in this study were approved by the Institutional Animal Care and Use Committee of CHA University. Four 7-week-old mice (ICR mice) (Orient Bio Co., Seongnam, South Korea) were housed separately in an animal resources facility, at a controlled temperature (20$22.8^{\circ} \mathrm{C}$ ) with a 12 -h light/12-h dark cycle. Chow and water were provided ad libitum.

\section{Tissue handling and implantation}

We used the patient-derived keloid xenograft mouse model to confirm the in vivo efficacy of rucaparib on keloid tissue. Immediately after surgical excision, a plastic surgeon deepithelialized human keloid tissue and evenly cut it into two pieces $\left(1.0 \times 1.0 \times 1.0 \mathrm{~cm}^{2}\right)$ with \#11 surgical blade; After immersion into Dulbecco's modified Eagle's medium (DMEM) solution, we implanted deepithelialized human keloid tissue into the ventral subcu- 
Table 1. Baseline demographics

\begin{tabular}{|c|c|c|c|c|c|c|}
\hline No. of Cases (n) & Age (years) & Gender & Skin type (Fitzpatrick) & Duration of Scar (years) & Scar Location & Cause \\
\hline 1 & 21 & $\mathrm{~F}$ & F III-IV & 7 & Abdomen & Spontaneous \\
\hline 2 & 56 & $\mathrm{~F}$ & F I-II & 30 & Chest wall & Infection \\
\hline 3 & 21 & $\mathrm{~F}$ & F III-IV & 7 & Axilla & Spontaneous \\
\hline 4 & 60 & $\mathrm{~F}$ & F I-II & 25 & Umbilicus & Surgery \\
\hline 5 & 37 & $\mathrm{~F}$ & F III-IV & 5 & Abdomen & Surgery \\
\hline 6 & 21 & $\mathrm{~F}$ & $\mathrm{~F}|-| \mid$ & 3 & Helix & Piercing \\
\hline 7 & 27 & $\mathrm{~F}$ & $\mathrm{~F} \mathrm{I-II}$ & 3 & Lobule & Piercing \\
\hline 8 & 23 & $\mathrm{~F}$ & $\mathrm{~F}|-| \mid$ & 4 & Lobule & Piercing \\
\hline 9 & 15 & $\mathrm{~F}$ & $\mathrm{~F}|-| \mid$ & 2 & Helix & Piercing \\
\hline
\end{tabular}

taneous pocket of mice of 7 weeks of age, and closed the wound with nylon 5-0. Appropriate dressing was done to minimize wound complications.

\section{In vivo explantation}

The animals were randomly assigned into two groups consisting of two mice each, depending on whether they will be injected with $P A R P 1$ inhibitor administration (experiment group), or without PARP1 inhibitor but with normal saline pretreatment (control group) 1 week afterward.

Seven days afterward, the experiment group $(n=2)$ was then treated with rucaparib $(1 \mathrm{mM}$, $0.5 \mathrm{~mL}$ ) by subdermal injection just around the implanted site. Meanwhile, the control group $(n=2)$ was treated with $0.5 \mathrm{~mL}$ of PBS.

Twelve weeks postoperatively, we harvested the implanted keloid tissue with sterile draping. We measured the dimensions along with documentation, the firmness of the tissue.

\section{Primary culture of keloid fibroblasts and NHDF}

The fibroblasts were isolated from the dermis by surgical excision. The dermis was cut into $\sim 5 \mathrm{~mm}^{3}$ pieces. The epidermis and lipid layer were removed with $2 \%$ dispase II (Sigma, St. Louis, MO) and the connective tissue was digested in $0.5 \mathrm{mg} / \mathrm{mL}$ collagenase A (Sigma) at $37^{\circ} \mathrm{C}$ for $3 \mathrm{~h}$ using a water bath. The digested solution was filtered through a $70 \mu \mathrm{m}$ strainer (BD Biosciences, San Diego, CA). The cell pellets were resuspended in, and washed with, $1 \times$ Dulbecco's phosphate-buffered saline (Gibco, Gaithersburg, MD). The cells were cultured in DMEM medium (Gibco) supplemented with $10 \%$ fetal bovine serum (FBS, Gibco) and 1\% penicillin/ streptomycin (Gibco) at $37^{\circ} \mathrm{C}$ and $5 \% \mathrm{CO}_{2}$. The medium was replaced every 2-3 days and the cells were subcultured at 70-80\% confluency. NHDF was also used as control. All experiments were performed with cells at passage 3 .

\section{Cell proliferation analysis (MTT assay)}

With the CellTiter 96 Aqueous One Solution Cell Proliferation Assay (Promega, Madison, WI), cell proliferation was measured on the basis of cellular conversion of the colorimetric reagent 3-(4,5dimethylthiazol-2-yl)-2,5-diphenyltetrazolium bromide (MTT) into soluble formazan by dehydrogenase enzyme found in metabolically proliferating cell. Following each treatment, $20 \mu \mathrm{L}$ of dye solution was added into each well in a 96-well plate and incubated for $2 \mathrm{~h}$. Subsequently, the absorbance was recorded at a wavelength of $490 \mathrm{~nm}$ using an enzyme-linked immunosorbent assay (ELISA) plate reader (Molecular Devices, Sunnyvale, CA).

\section{Migration assay}

The chemotactic migration of fibroblasts was measured with a transwell migration apparatus. Conditioned medium derived from the culture of keloid or normal fibroblasts was added to the lower wells of the transwell chamber. Fibroblasts were trypsinized, resuspended in serum-free DMEM at a concentration of $1.2 \times 10^{4}$ or $2 \times 10^{4}$ cells $/ \mathrm{mL}$, and added into the upper wells of the transwell chamber with a filter with $8-\mu \mathrm{m}$ pores. The chambers were incubated for $12 \mathrm{~h}$, and the interior of the inserts was gently swabbed to remove nonmigratory cells. Gentle washing of the transwell for 2 min in $1 \times \mathrm{PBS}$ was performed. The transwell was placed into a $4 \%$ paraformaldehyde solution for $15 \mathrm{~min}$, before washing with $1 \times \mathrm{PBS}$. The insert was transferred to a clean well containing $700 \mu \mathrm{L}$ of $0.1 \%$ Crystal Violet and incubated for $30 \mathrm{~min}$ at room temperature. Crystal Violet stain was removed and washed twice by PBS. Each insert was transferred to an empty well, and $200 \mu \mathrm{L}$ of $100 \%$ acetic acid per well was added. Then, $100 \mu \mathrm{L}$ from each sample was transferred to a 96-well plate and was measured by the OD $560 \mathrm{~nm}$ in a plate reader.

\section{Immunoblot analysis}

Cells were lysed for 20 min on ice with lysis buffer and centrifuged at $14,000 \mathrm{~g}$ for $10 \mathrm{~min}$ at $4^{\circ} \mathrm{C}$. Samples were resolved by sodium dodecyl sulfate/polyacrylamide gel electrophoresis, trans- 
ferred to nitrocellulose membranes, blotted with appropriate primary antibodies at a dilution of 1:1000, and treated with peroxidase-conjugated secondary antibodies (Biosource International, Camarillo, CA). We also performed electrophoresis of protein extracts derived from keloid or normal dermal tissue using a Tris-glycine buffer system, and subsequent blottings were performed. Bound antibodies were visualized using chemiluminescent substrate (ECL; Amersham, Arlington Heights, IL) and exposed to Kodak X-OMAT film (Kodak, New Haven, CT). Primary antibody for rabbit anti-PARP1 was purchased from Cell Signaling Technology (Danvers, MA). Goat anti-actin antibody was from Santa Cruz Biotechnology, Inc. (Santa Cruz, CA). Densitometric analyses were performed with ImageJ software (National Institutes of Health, Bethesda, MD).

\section{Real time-polymerase chain reaction analysis}

Total ribonucleic acids (RNAs) were extracted from keloid fibroblasts using TRIzol Reagent (Invitrogen, Carlsbad, CA). Complementary deoxyribonucleic acid (cDNA) templates were prepared using oligo(dT) random primers and Moloney Murine Leukemia Virus (MoMLV) reverse transcriptase. After the reverse transcription reaction, the cDNA template was amplified by polymerase chain reaction (PCR) using Taq polymerase (Invitrogen). PARP1 was quantitated by real-time PCR (LightCycler; Roche Molecular Biochemicals, Mannheim, Germany) using SYBR Green as the fluorophore (Molecular Probes, Eugene, OR). Primers of PARP1 were as follows: forward: $5^{\prime}$-gga gtggatgaagtggcgaa-3'; and reverse: $5^{\prime}$-ggcgatcttg gaccgagtag-3'. Glyceraldehyde-3-phosphate dehydrogenase (GAPDH) gene expression was used as a control. The level of PARP1 mRNA expression was calculated as the relative intensity of the PCR product bands compared with that from the GAPDH gene using the $2^{-\Delta \Delta \mathrm{Ct}}$ method. The mRNA expressions of TGF- $\beta$, and matrix metallopeptidases (MMPs) were also assessed. All PCR experiments were performed in triplicate.

\section{Statistical analyses}

Statistical analyses were performed using PASW version 21.0 (SPSS, Inc., Chicago, IL). All experimental results were obtained from three independent experiments using cells from three separate isolations and are presented as mean \pm standard deviation (SD). For comparisons between groups, data were analyzed by the Mann-Whitney U test or one-way ANOVA. For all tests, $p<0.05$ was regarded as statistically significant.

\section{RESULTS}

\section{Patient demographics}

A total of nine patients with eight keloids were achieved. Table 1 shows the baseline demographics of patients included in this study. All patients were female with mean age of 31.2. Six patients had skin type F I-II and other three patients had F III-IV. The duration of scar ranged between $2-30$ years. The fibroblasts were isolated from all patients' scar, and then used for all following in vitro analyses.

\section{The protein expressions of PARP1 and smad3 were enhanced in keloid tissue as compared with normal dermal tissue}

First, we evaluated the protein expressions of $P A R P 1$, smad3, and phosphorylated smad3 in keloid tissue, which are the key signaling pathways in forming a keloid. As shown in Figure 1A, the protein expressions of PARP1, smad3, and phosphorylated smad3 were significantly enhanced in keloid tissue as compared with normal dermal tissue.

\section{The effect of rucaparib on the expression of PARP1 in keloid fibroblasts}

The expression of PARP1 mRNA on keloid cell was significantly suppressed by rucaparib treatment $(20 \mu \mathrm{M})$, whereas that on NHDF was not suppressed by rucaparib treatment (Fig. $1 \mathrm{~B} ; p<0.05$ ). We also analyzed the effect of rucaparib on the expression $P A R P 1$ protein. Rucaparib treatment $(20 \mu \mathrm{M})$ significantly attenuated the expression of $P A R P 1$ protein as shown in Figure 1C.

\section{The therapeutic efficacy of rucaparib in keloid fibroblasts}

Then, we evaluated whether rucaparib \pm triamcinolone suppress proliferation of keloid fibroblasts. Keloid cell growth following treatment with rucaparib at various concentrations $(0,2,10$, $20 \mu \mathrm{M}$ ) was evaluated by MTT assays. As shown in Figure 2A, rucaparib $(20 \mu \mathrm{M})$ significantly suppressed the proliferation of keloid fibroblasts. Moreover, the combination of rucaparib $(20 \mu \mathrm{M})$ and triamcinolone $(50 \mu \mathrm{M})$ showed additive suppressive effect on keloid fibroblasts as compared with rucaparib single therapy (Fig. 2B).

\section{Rucaparib significantly decreased migration of keloid fibroblasts}

We compared the migration ability of keloid fibroblasts using the transwell assay. In comparison with the untreated fibroblasts, those treated with rucaparib $(10 \mu \mathrm{M}$; the concentration that does not significantly suppress the proliferation of keloid cells) showed attenuated migration ability in both concentrations of $1.2 \times 10^{4}$ and $2 \times 10^{4}$ cells/mL (Fig. 3). 
A

\section{Keloid tissue vs. normal tissue}

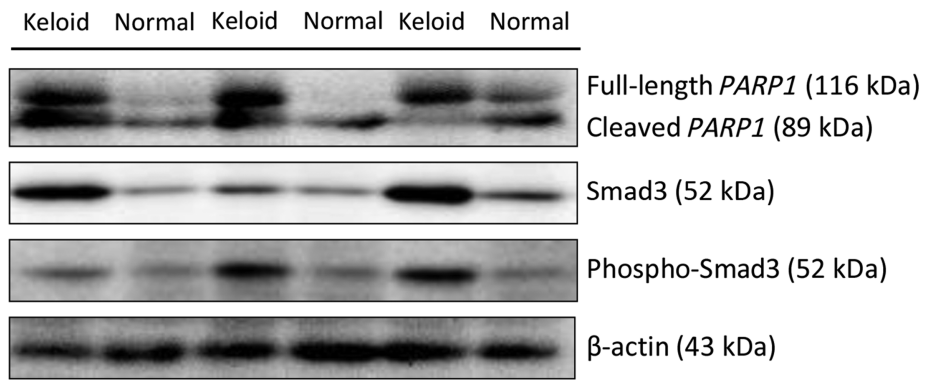

B

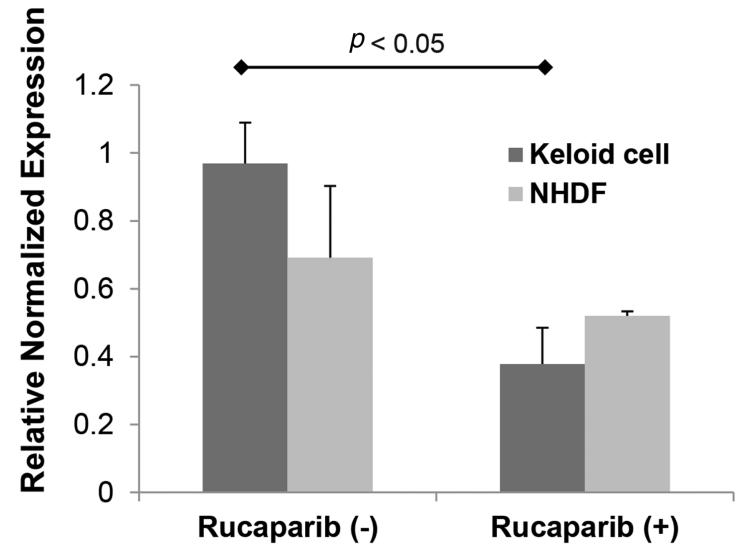

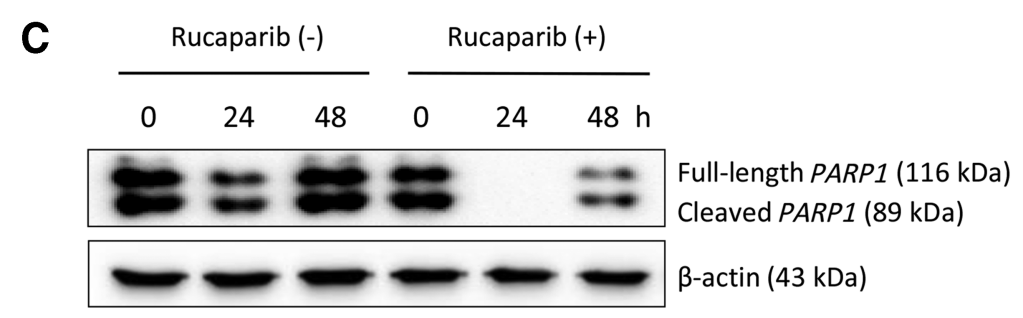

Figure 1. Rucaparib inhibited the expression of $P A R P 1$ in keloid fibroblasts. (A) Immunoblot analyses revealed that the protein expressions of $P A R P 1$, smad3, and phosphorylated-smad3 were enhanced in keloid tissue as compared with normal dermal tissue. (B) Real-time PCR revealed that rucaparib treatment $(20 \mu \mathrm{M})$ significantly decreased PARP1 mRNA expression on keloid fibroblasts normalized to GAPDH expression levels. The experiments were repeated nine times. (C) Immunoblot analyses revealed that PARP1 protein expression was suppressed by rucaparib treatment (20 $\mu \mathrm{M})$. GAPDH, glyceraldehyde-3-phosphate dehydrogenase; NHDF, normal human dermal fibroblasts; PARP1, poly(ADP-ribose) polymerase 1.

A

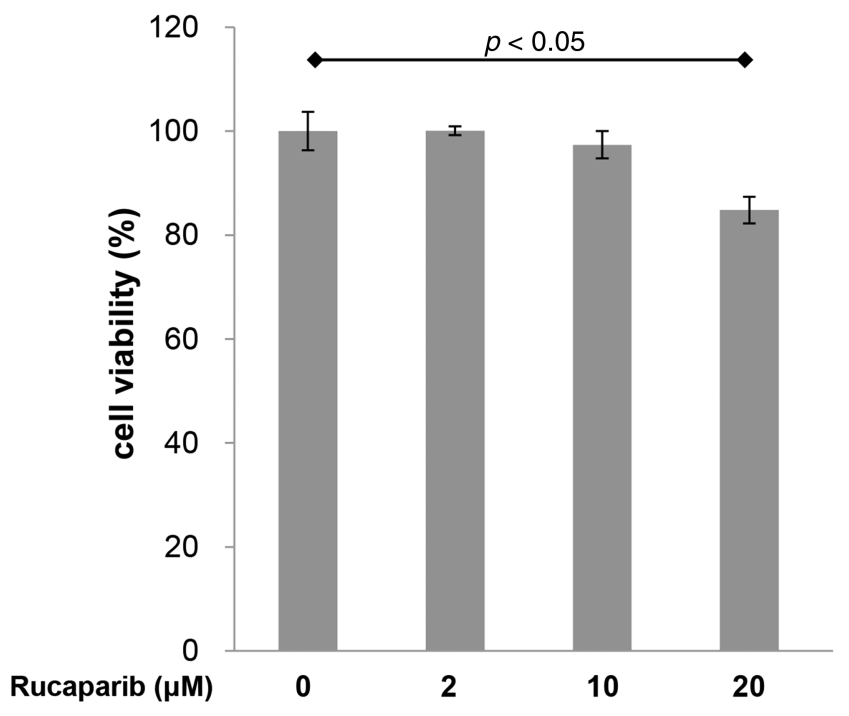

B

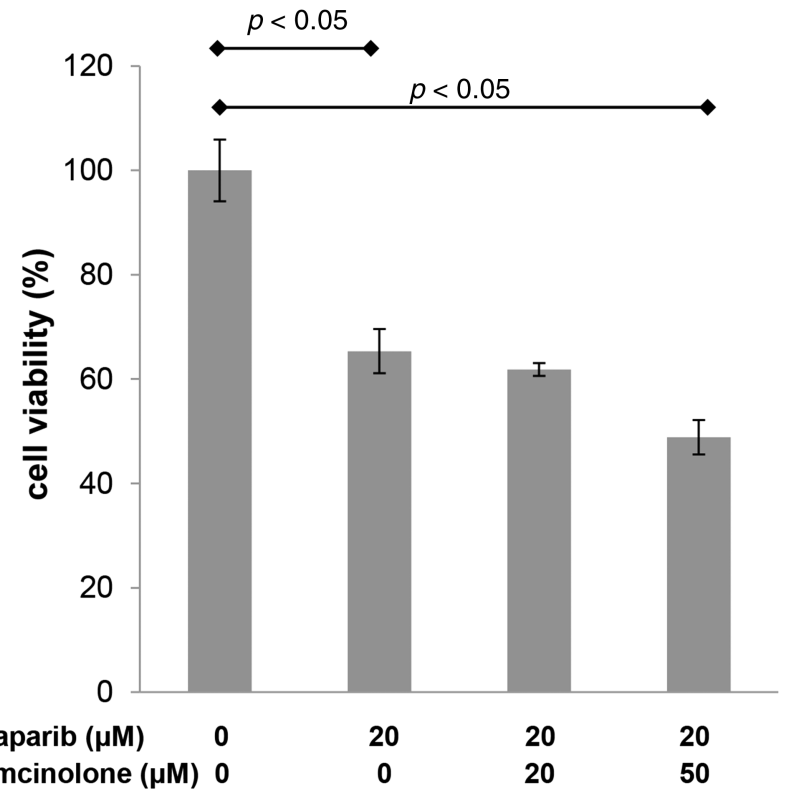

Figure 2. The effect of rucaparib on proliferation of keloid fibroblasts. (A) Keloid cell growth following treatment with rucaparib at various concentration $(0,2$, $10,20 \mu \mathrm{M}$ ) was evaluated by MTT assays. Data are expression as mean standard deviation of percent changes of triplicate optical densities. (B) Rucaparib $(20 \mu \mathrm{M})$ significantly decreased proliferation of keloid fibroblasts. The combination of rucaparib $(20 \mu \mathrm{M})$ and triamcinolone $(50 \mu \mathrm{M})$ showed additive suppressive effect on keloid fibroblasts as compared with rucaparib single therapy. Data are expression as mean standard deviation of percent changes of triplicate optical densities. MTT, 3-(4,5-dimethylthiazol-2-yl)-2,5-diphenyltetrazolium bromide. 


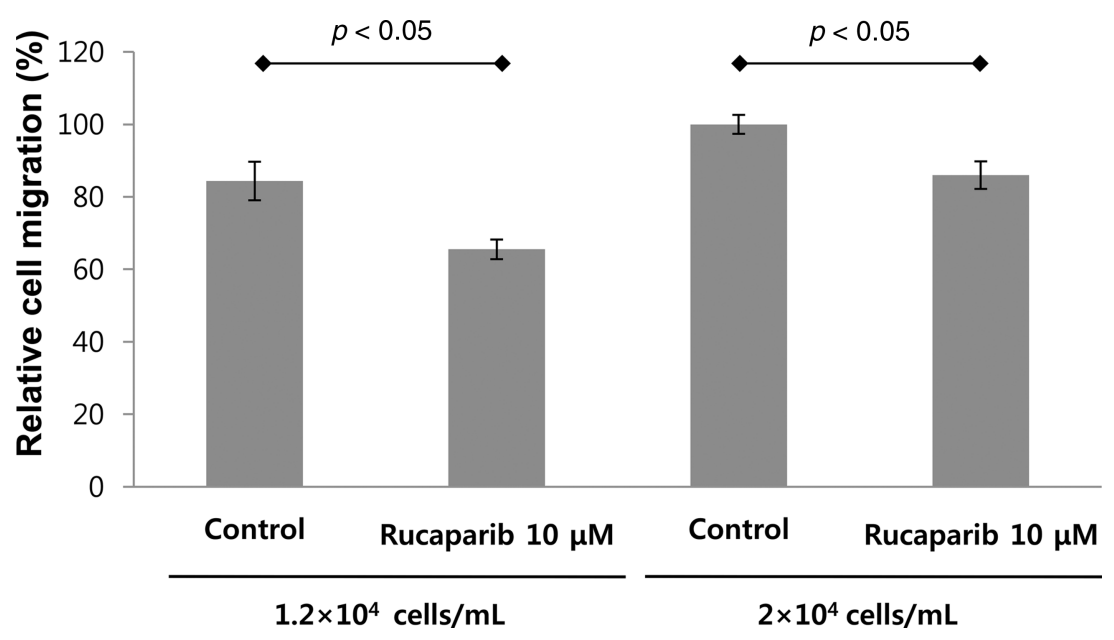

Figure 3. Rucaparib suppressed migration of keloid fibroblasts. The migration activity of dermal fibroblasts was decreased following rucaparib treatment $(10 \mu \mathrm{M})$. The migration of the cells was analyzed by the transwell assay.

\section{Rucaparib significantly suppressed}

\section{the fibrosis markers in keloid fibroblasts}

We finally evaluated whether rucaparib reduces the expression of fibrosis markers in keloid cells. The mRNA expression of fibrosis markers, including MMP-1, $-2,-3$, and $-9, \alpha$-smooth muscle actin, fibronectin, and connective tissue growth factor was significantly attenuated in keloid fibroblasts after rucaparib treatment $(20 \mu \mathrm{M})$ (Fig. 4)

\section{Rucaparib significantly suppressed} the fibrosis markers in keloid fibroblasts

We evaluated whether rucaparib reduces the fibrosis in keloid xenograft model. According to twodimensional measurements, rucaparib significantly reduced its size along with relative softness compared with the control group (Fig. 5). The relative size of explanted keloid tissue in the experimental group is 55\% compared with the control group.

\section{Rucaparib significantly reduced the dimension of keloid tissue in patient-derived keloid xenograft model}

We implanted the same-sized deepithelialized human keloid tissue $(1 \times 1 \times 1 \mathrm{~cm})$ into the subcutaneous pocket of 7 weeks old ICR mice $(n=4)$, and closed the wound. After 7 days, rucaparib $(1 \mathrm{mM}, 0.5 \mathrm{~mL})$ or PBS $(0.5 \mathrm{~mL})$ was injected subdermally just around the implanted site. At the time of postoperative 12 weeks, the mean dimension of keloid tissue of ruca-

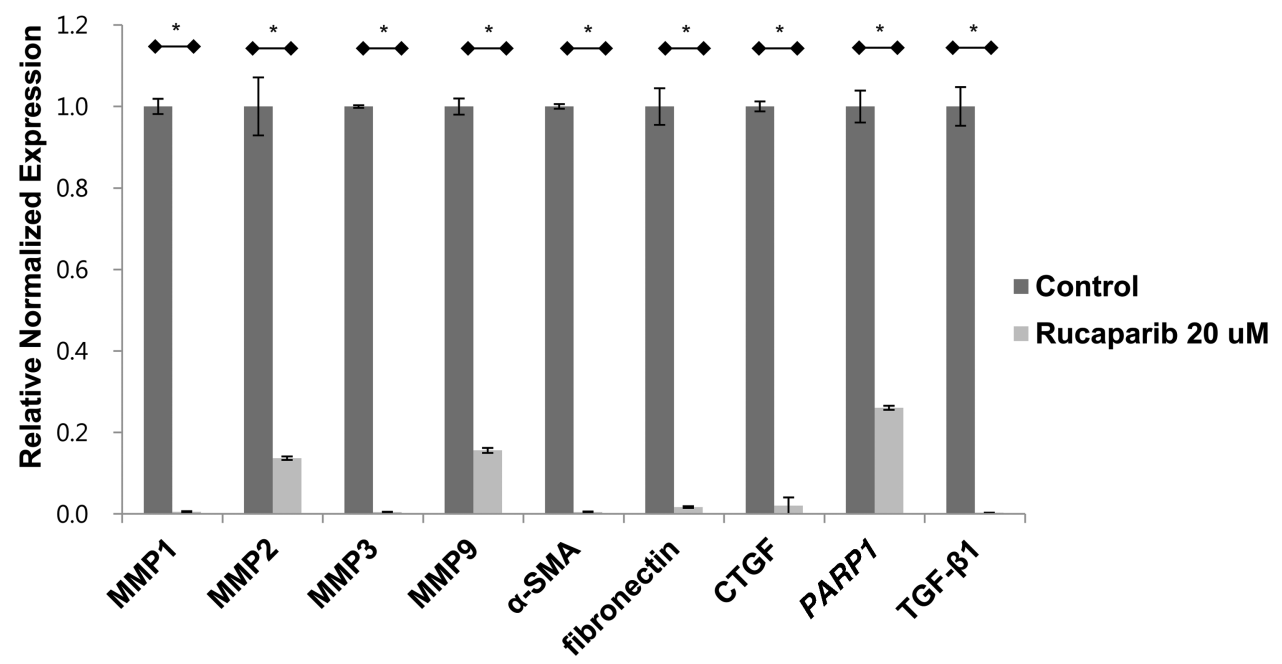

Figure 4. Rucaparib attenuated the expression of fibrosis markers in keloid fibroblasts. Real-time PCR revealed that rucaparib treatment (20 $\mu \mathrm{M})$ significantly decreased the mRNA expressions of MMP-1, 2, 3, 9, and $\alpha$-SMA, fibronectin, and CTGF on keloid fibroblasts normalized to GAPDH expression levels. ${ }^{*} p<0.05$. CTGF, connective tissue growth factor; MMP, matrix metallopeptidase; PCR, polymerase chain reaction; SMA, smooth muscle actin; TGF- $\beta 1$, transforming growth factor-beta 1. 

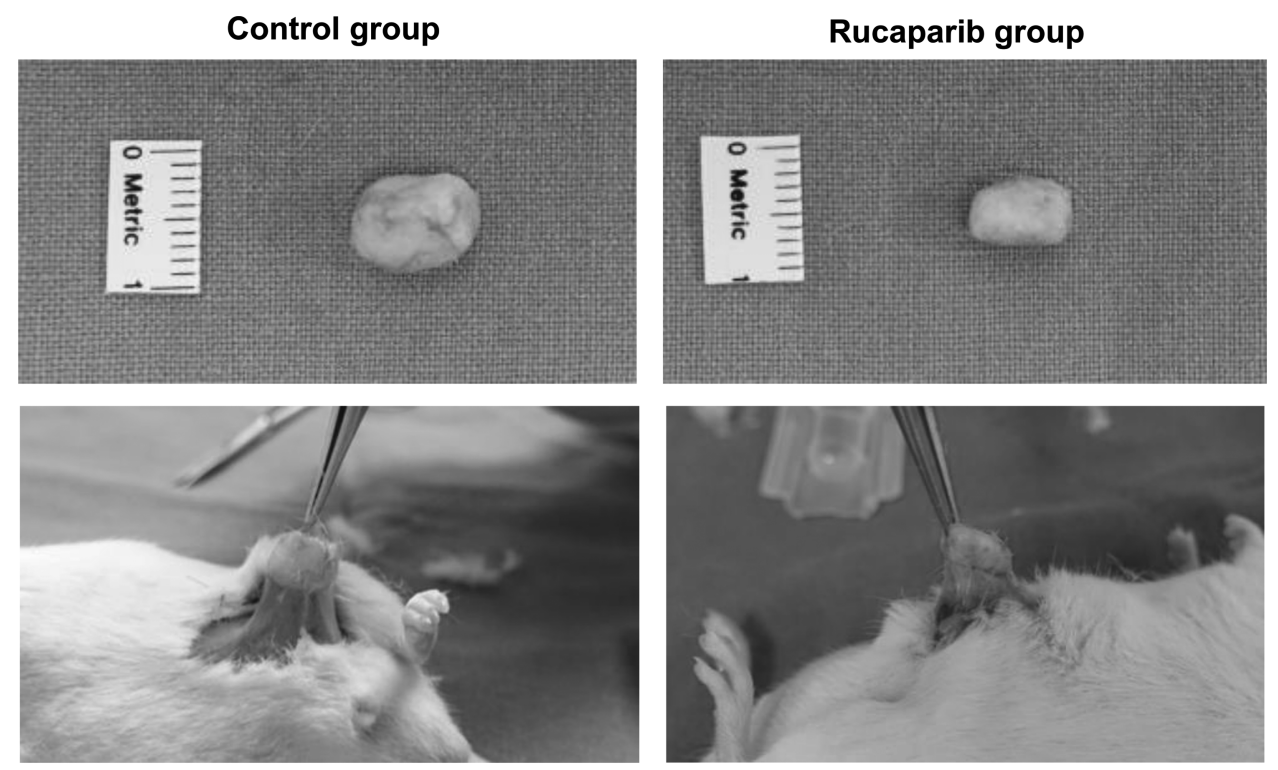

Figure 5. Rucaparib decreased the dimension of implanted keloid tissue in keloid xenograft model. Rucaparib significantly reduced the dimension of patientderived implanted keloid tissue after 12 weeks as compared with control.

parib group was $0.9 \times 0.5 \mathrm{~cm}$, whereas that of control group was $1 \times 1 \mathrm{~cm}$ (Fig. 5). Moreover, the implanted tissue in rucaparib group had much less adhesion with subcutaneous pocket compared with that in control group. We also measured the degree of firmness of the mass manually, which revealed five out of five in the control group, whereas two out of five in the rucaparib group.

\section{DISCUSSION}

Keloids, which occur because of unbalanced homeostasis in normal wound healing, do not regress spontaneously and frequently recur after surgical excision alone. The development of keloid scars involves a complicated multistep process that is likely driven by many different genetic, environmental, and local mechanophysiological factors. To date, no theory of the general mechanisms of pathologic scar formation has provided a full explanation of the clinicopathological characteristics of keloid.

$P A R P-1$ is known to affect the transcriptional output of a Smad-sensitive promoter, which suggests that $P A R P-1$ may play a more fundamental role in negatively regulating expression of TGF- $\beta$ responsive genes. ${ }^{28}$ Functionally, $P A R P-1$ is known to regulate transcription factors, such as $\mathrm{p} 53, \mathrm{Sp} 1$, and NF- $\kappa \mathrm{B} .{ }^{29}$ In addition, $\mathrm{p} 53,{ }^{30} \mathrm{Sp} 1,{ }^{31}$ and NF-kB ${ }^{32}$ is also well known as one of the factors involved in keloid pathogenesis. In addition, $P A R P 1$ is known to prerequisite for TGF- $\beta 1$-induced Smad3 activation in rat vascular smooth muscle cells. These series of molecular findings implies that targeting PARP1 may be a promising therapeutic target against keloid diseases characterized by dysregulated TGF- $\beta$ / Smad3 pathway.

On the other hand, PARP1 has been in the recent spotlight due to its strong relation with fibrotic disease in various organs. In cardiac tissue, $P A R P-1$ inhibition partially decreased autophagy, abrogated cardiac fibrosis, and significantly improved cardiac function post-MI. ${ }^{33}$ In renal tissue, PARP enzyme inhibition alleviates diabetic nephropathy through decreasing inflammation, oxidative stress, and renal fibrosis in diabetic animals. ${ }^{34}$ In liver, PARP inhibition protects against alcoholic and nonalcoholic steatohepatitis by attenuating hepatic triglyceride accumulation, metabolic dysregulation, or inflammation and/or fibrosis in models of nonalcoholic steatohepatitis. ${ }^{35}$ PARP1 loss or downregulation itself alters the expression of many genes involved in cell cycle control and stress response, especially p53. ${ }^{22}$

Despite recent progress in recognizing its important role of PARP activation in fibrotic disease, however, no study unveiling its possible role in skin fibrosis, including keloid disease, has yet been done.

So, we initiated this study by analyzing baseline $P A R P 1$ expression in normal human dermal tissue and keloid tissue. To our expectation, $P A R P 1$ was highly expressed in keloid tissue compared with normal human dermal tissue. We also revealed that PARP1 inhibition significantly inhibits the expression of keloid fibroblast in terms of cell viability, cell migration, and fibrosis marker expression. We performed an in vivo study to increase clinical significance and successfully demonstrated 
that PARP1 inhibitor rucaparib, the FDAapproved chemotherapeutic drug for ovarian cancer, significantly decreased the degree of fibrosis in patient-derived keloid xenograft model. Conclusively, these findings suggested that $P A R P 1$ inhibition can be a potential therapeutic target for keloid treatment and insinuated rucaparib can be a successful option for the treatment of keloid disease.

We also investigated TGF- $\beta$ expression according to the delivered drugs. Even though both TA and PARP1 inhibitors significantly decreased TGF- $\beta$ expression, PARP1 inhibitors showed a sharper decrease revealing an intimate relationship between PARP1 and TGF- $\beta$. In addition, we think that PARP1 inhibitors can be added to traditional TA to increase therapeutic efficacy based on our study results. However, this concept should be further studied in a clinical setting to optimize dose to be delivered and its composition.

The PARP1 inhibitor, rucaparib, is a recently FDA-approved therapeutic agent for ovarian cancer. This is the first study reporting that PARP is overexpressed in keloid disease and that pharmacological inhibition with rucaparib significantly attenuates the expression of fibrosis markers in vitro and in vivo. Altogether, PARP1 inhibition might be a promising therapeutic strategy for the treatment of keloid disease.

\section{INNOVATION}

The study data suggest that $P A R P 1$ might be a novel therapeutic target for keloid disease. PARP1 inhibitor, rucaparib, might be a promising therapeutic drug for the treatment of keloid disease.

\section{ACKNOWLEDGMENTS AND FUNDING SOURCES}

This work was supported by the National Research Foundation of Korea (NRF) grant funded by the Korea government (MSIP; Ministry of Science, ICT \& Future Planning) (No. 2017R1C1B5017180 and 2017R1B5A2085456 to T.H.P., and No. 2017R 1C1B5076045 to Y.J.P.).

\section{AUTHOR DISCLOSURE AND GHOSTWRITING}

The authors declare that no financial interests exist. This article was written by the authors listed. No ghostwriters were used to write this article.

\section{ABOUT THE AUTHORS}

Tae Hwan Park, MD, PhD is working as an assistant professor at the Department of Plastic and Reconstructive surgery, CHA Bundang Medical Center, Korea. He is currently affiliated with Georgetown University, Washington D.C. as a visiting scholar. Yuri Cho, MD, PhD, co-corresponding author of this article, is working as an assistant professor at the Department of Internal Medicine, CHA Gangnam Medical Center, Korea. She has interests in cancer treatment and neoplastic diseases. Chan Woo Kim, MD is a chief resident working with Dr. Park TH. Jin Sik Choi, MD, who is a research associate with Dr. Park TH, and Yun Joo Park, MD, who is working as an assistant professor at the Department of Radiology in Hallym University Sacred Heart Hospital, Korea, have collaborated this project with Dr. Park TH. Yosep Chong, MD, who is working as an assistant professor at the Department of Pathology in Catholic University, St. Mary's Hospital, Korea, have collaborated this project with Dr. Park TH Min Ji Park, MSc, who is a full-time researcher working at CHA Gangnam Medical Center with Dr. Cho Y.

\section{REFERENCES}

1. Unahabhokha T, Sucontphunt A, Nimmannit U, Chanvorachote $P$, Yongsanguanchai $N$, Pongrakhananon V. Molecular signalings in keloid disease and current therapeutic approaches from natural based compounds. Pharm Biol 2015;53:457-463

2. Shih B, Garside E, McGrouther DA, Bayat A Molecular dissection of abnormal wound healing processes resulting in keloid disease. Wound Repair Regen 2010;18:139-153.

3. Lim CP, Phan TT, Lim IJ, Cao X. Stat3 contributes to keloid pathogenesis via promoting collagen production, cell proliferation and migration. Oncogene 2006;25:5416-5425.
4. Li Y, Liu H, Liang Y, Peng P, Ma X, Zhang X. DKK3 regulates cell proliferation, apoptosis and collagen synthesis in keloid fibroblasts via TGF-beta1/ Smad signaling pathway. Biomed Pharmacother 2017:91:174-180.

5. Sun Q, Guo S, Wang CC, Sun X, Wang D, Xu N, et al. Cross-talk between TGF-beta/Smad pathway and 
Wnt/beta-catenin pathway in pathological scar formation. Int J Clin Exp Pathol 2015;8:7631-7639.

6. Yao X, Cui X, Wu X, Xu P, Zhu W, Chen X, et al. Tumor suppressive role of miR-1224-5p in keloid proliferation, apoptosis and invasion via the TGFbeta1/Smad3 signaling pathway. Biochem Biophys Res Commun 2018;495:713-720.

7. Zhao B, Guan H, Liu JQ, Zheng Z, Zhou Q, Zhang $J$, et al. Hypoxia drives the transition of human dermal fibroblasts to a myofibroblast-like phenotype via the TGF-beta1/Smad3 pathway. Int J Mol Med 2017:39:153-159.

8. Ghazizadeh M, Tosa M, Shimizu H, Hyakusoku H, Kawanami O. Functional implications of the IL-6 signaling pathway in keloid pathogenesis. J Invest Dermatol 2007;127:98-105

9. Quong WL, Kozai Y, Ogawa R. A case of keloids complicated by castleman's disease: interleukin-6 as a keloid risk factor. Plast Reconstr Surg Global Open 2017:5:e1336.

10. Tosa M, Ghazizadeh M, Shimizu H, Hirai T, Hyakusoku $\mathrm{H}$, Kawanami 0 . Global gene expression analysis of keloid fibroblasts in response to electron beam irradiation reveals the involvement of interleukin-6 pathway. J Invest Dermatol 2005; 124:704-713.

11. Tosa M, Watanabe A, Ghazizadeh M. IL-6 polymorphism and susceptibility to keloid formation in a Japanese population. J Invest Dermatol 2016: 136:1069-1072

12. Uitto J. IL-6 signaling pathway in keloids: a target for pharmacologic intervention? J Invest Dermatol 2007;127:6-8

13. Zhu XJ, Li WZ, Li H, Fu CQ, Liu J. Association of interleukin-6 gene polymorphisms and circulating levels with keloid scars in a Chinese Han population. Genet Mol Res 2017;16.

14. Hu ZC, Tang B, Guo D, Zhang J, Liang YY, Ma D, et al. Expression of insulin-like growth factor-1 receptor in keloid and hypertrophic scar. Clin Exp Dermatol 2014;39:822-828.

15. Romero-Valdovinos M, Cardenas-Mejia A, Gutierrez-Gomez C, Flisser A, Kawa-Karasik S, Ortiz-Monasterio F. Keloid skin scars: the influence of hyperbaric oxygenation on fibroblast growth and on the expression of messenger RNA for insulin like growth factor and for transforming growth factor. In Vitro Cell Dev Biol Anim 2011;47:421-424.
16. Kim J, Park JC, Lee MH, Yang CE, Lee JH, Lee WJ. High-mobility group box 1 mediates fibroblast activity via RAGE-MAPK and NF-kappaB signaling in Keloid scar formation. Int J Mol Sci 2017;19: pii: E76.

17. Wang W, Qu M, Xu L, Wu X, Gao Z, Gu T, et al. Sorafenib exerts an anti-keloid activity by antagonizing TGF-beta/Smad and MAPK/ERK signaling pathways. J Mol Med 2016;94:1181-1194.

18. Park TH, Rah DK, Chang CH, Kim SY. Establishment of patient-derived keloid xenograft model. J Craniofac Surg 2016;27:1670-1673.

19. Shang T, Yao B, Gao D, Xie J, Fu X, Huang S. A novel model of humanised keloid scarring in mice. Int Wound J 2018;15:90-94.

20. Sunaga A, Kamochi H, Sarukawa S, Uda H, Sugawara $Y$, Asahi R, et al. Reconstitution of human keloids in mouse skin. Plast Reconstr Surg Global Open 2017;5:e1304.

21. Jagtap P, Szabó C. Poly (ADP-ribose) polymerase and the therapeutic effects of its inhibitors. Nat Rev Drug Discov 2005;4:421.

22. Rouleau M, Patel A, Hendzel MJ, Kaufmann SH, Poirier GG. PARP inhibition: PARP1 and beyond. Nat Rev Cancer 2010;10:293.

23. Sodhi RK, Singh N, Jaggi AS. Poly (ADP-ribose polymerase-1 (PARP-1) and its therapeutic implications. Vasc Pharmacol 2010;53:77-87.

24. Lucarini L, Durante M, Lanzi C, Pini A, Boccalini G, Calosi L, et al. HYDAMTIQ, a selective PARP-1 inhibitor, improves bleomycin-induced lung fibrosis by dampening the TGF- $\beta /$ SMAD signalling pathway. J Cell Mol Med 2017;21:324-335.

25. Park TH, Seo SW, Kim J-K, Chang CH. Outcomes of surgical excision with pressure therapy using magnets and identification of risk factors for recurrent keloids. Plast Reconstr Surg 2011;128:431-439.

26. Akita S, Akino K, Yakabe A, Imaizumi T, Tanaka K, Anraku $K$, et al. Combined surgical excision and radiation therapy for keloid treatment. J Craniofac Surg 2007;18:1164-1169.

27. Ud-Din S, Thomas G, Morris J, Bayat A. Photodynamic therapy: an innovative approach to the treatment of keloid disease evaluated using subjective and objective non-invasive tools. Arch Dermatol Res 2013;305:205-214.

28. Lonn $P$, van der Heide LP, Dahl M, Hellman U, Heldin CH, Moustakas A. PARP-1 attenuates Smadmediated transcription. Mol Cell 2010;40:521-532.
29. Kraus WL. Transcriptional control by PARP-1: chromatin modulation, enhancer-binding, coregulation, and insulation. Curr Opin Cell Biol 2008; 20:294-302.

30. Heitzer E, Seidl H, Bambach I, Schmidbauer U, Cerroni L, Wolf P. Infrequent p53 gene mutation but UV gradient-like p53 protein positivity in keloids. Exp Dermatol 2012;21:277-280.

31. Mukhopadhyay A, Khoo A, Cheong HH, Chan SY Aalami 0, Lim IJ, et al. Targeting of Sp1 transcription factor: a novel therapeutic approach for keloids, an in vitro analysis. Exp Dermatol 2007; 16:1023-1031.

32. Messadi DV, Doung HS, Zhang Q, Kelly AP, Tuan $T L$, Reichenberger E, et al. Activation of NFkappaB signal pathways in keloid fibroblasts. Arch Dermatol Res 2004;296:125-133.

33. Wang $H$, Yang $X$, Yang $\mathrm{O}$, Gong $L, X u H$, Wu Z. PARP-1 inhibition attenuates cardiac fibrosis induced by myocardial infarction through regulating autophagy. Biochem Biophys Res Commun 2018; 503:1625-1632.

34. Zakaria EM, El-Maraghy NN, Ahmed AF, Ali AA El-Bassossy HM. PARP inhibition ameliorates nephropathy in an animal model of type 2 diabetes: focus on oxidative stress, inflammation, and fibrosis. Naunyn Schmiedebergs Arch Pharmacol 2017;390:621-631.

35. Mukhopadhyay P, Horvath B, Rajesh M, Varga ZV Gariani $K$, Ryu D, et al. PARP inhibition protects against alcoholic and non-alcoholic steatohepatitis. J Hepatol 2017;66:589-600.

\section{Abbreviations Used}

CTGF $=$ connective tissue growth factor

DMEM = Dulbecco's modified Eagle's medium

$F B S=$ fetal bovine serum

GAPDH = glyceraldehyde-3-phosphate dehydrogenase

$\mathrm{MMP}=$ matrix metallopeptidase

MTT $=3$-(4,5-dimethylthiazol-2-y)-2,5diphenyltetrazolium bromide

$\mathrm{NHDF}=$ normal human dermal fibroblasts

PARP1 $=$ poly(ADP-ribose) polymerase 1

$\mathrm{PCR}=$ polymerase chain reaction

$\mathrm{SD}=$ standard deviation

SMA $=$ smooth muscle actin

TGF- $\beta 1=$ transforming growth factor-beta 1 